\title{
Changes in sinonasal mucosa in Wegener granulomatosis
}

\section{Chao-Yin Kuo MD, Chih-Hung Wang MD PhD}

Competing interests: None declared.

This article has been peer reviewed.

Correspondence to: Chih-Hung Wang, chw@ms3.hinet.net

\section{CMAJ 2014. DOI:10.1503} /cmaj.131881

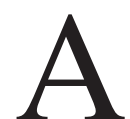
63-year-old woman presented with a twomonth history of intermittent epistaxis. In addition, she reported a sensation of fullness and progressive hearing loss in her left ear over the previous two weeks. Our differential diagnosis for these unexplained symptoms of the upper airway focused on inflammatory diseases or a neoplasm involving the sinonasal tract and nasopharynx. Nasal endoscopy showed friable erythematous and granulomatous mucosa over the right middle turbinate and left inferior turbinate (Figure 1A). Otoscopy showed an effusion of the left middle ear. The patient's C-reactive protein level was high, and
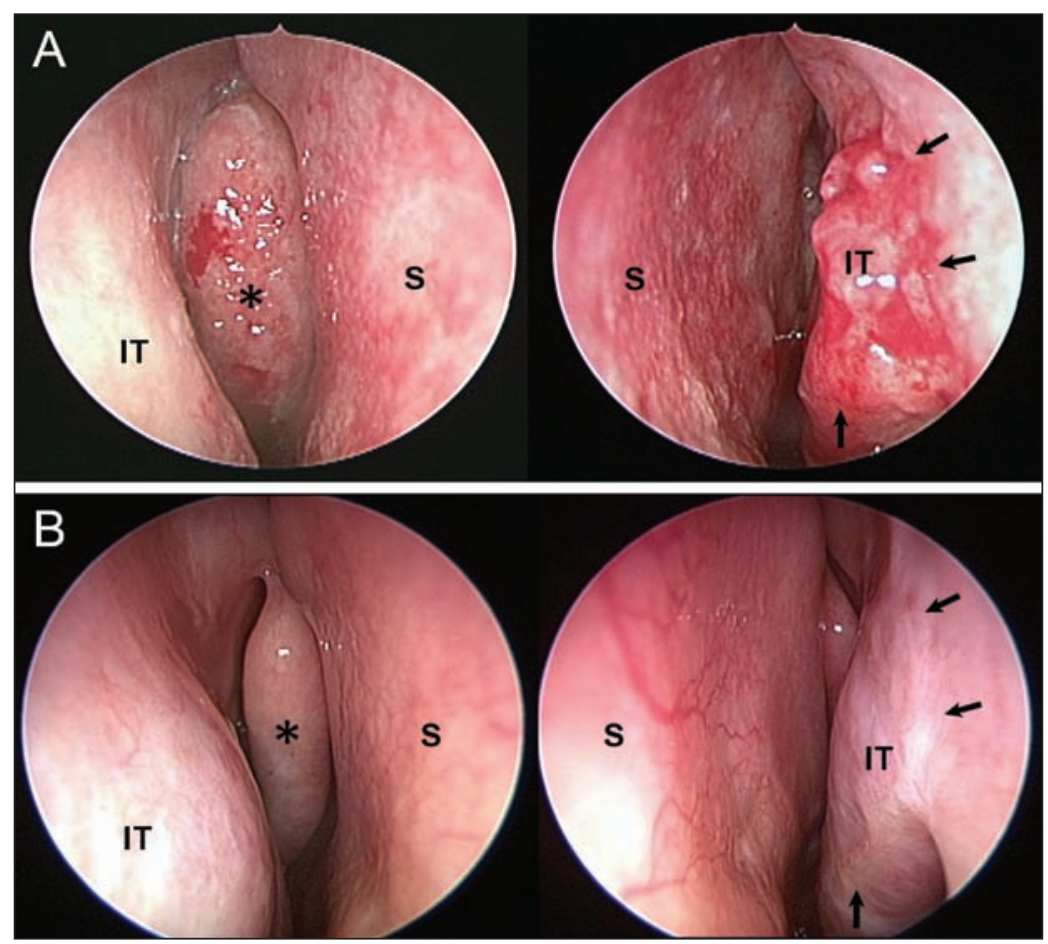

Figure 1: Changes in sinonasal mucosa before and after treatment in a 63-year-old woman with Wegener granulomatosis. (A) Sinoscopic examination at presentation showed friable erythematous and granular mucosa over the right middle turbinate (*) and the left inferior turbinate (arrows). (B) After eight months of treatment, a sinoscopic examination showed mucosal recovery of the right middle turbinate $(*)$ and the left inferior turbinate (arrows). $\mathrm{S}=$ septum, IT = inferior turbinate. an antineutrophilic cytoplasmic antibody assay was positive for antiproteinase 3 . Histopathologic examination of a biopsy of the turbinate confirmed a diagnosis of Wegener granulomatosis.

The patient was given rituximab and methylprednisolone intravenously, followed by maintenance treatment with azathioprine, hydroxychloroquine and prednisolone given orally. After eight months of treatment, her condition had improved, the effusion of her middle ear had disappeared, laboratory tests showed a decrease in her levels of antiproteinase 3 and C-reactive protein, and her nasal lesions had largely resolved (Figure 1B).

Although Wegener granulomatosis represents a general inflammation of blood vessels and may involve multiple organ systems, up to $95 \%$ of patients will present with otorhinolaryngologic involvement as the first sign of the disease. ${ }^{1}$ These patients may present with epistaxis, sinusitis, nasal disease or hearing loss. ${ }^{2}$ Granular, irregular thickening and crusting of the mucosa are characteristic sinonasal findings in Wegener granulomatosis, but they may be present in other granulomatous diseases such as sarcoidosis and Churg-Strauss syndrome. ${ }^{1}$ In patients with clinical features suggestive of Wegener granulomatosis, nasal endoscopy may show typical mucosal changes, and a biopsy will confirm the diagnosis if necrotizing granulomatous vasculitis is observed ${ }^{2}$ (Appendix 1, available at www.cmaj.ca/lookup /suppl/doi:10.1503/cmaj.131881/-/DC1).

\section{References}

1. Gottschlich S, Ambrosch P, Kramkowski D, et al. Head and neck manifestations of Wegener's granulomatosis. Rhinology 2006; 44:227-33.

2. Haris M, Koulaouzidis A, Yasir M, et al. Wegener's granulomatosis. CMAJ 2008;178:25-6.

Affiliations: Department of Otorhinolaryngology-Head and Neck Surgery (Kuo, Wang), Tri-Service General Hospital, National Defense Medical Center, Taipei, Taiwan; Graduate Institute of Medical Sciences (Wang), National Defense Medical Center, Taipei, Taiwan 